\title{
A Review on Photonic Crystal Logic Gates
}

\author{
Luis E. Pedraza Caballero ${ }^{1}$ and Omar P. Vilela Neto ${ }^{1}$ \\ ${ }^{1}$ Department of Computer Science, Universidade Federal de Minas Gerais, Belo Horizonte, 31270-901, Brazil \\ e-mail: omar@dcc.ufmg.br
}

\begin{abstract}
We review the most important techniques to build logic devices in photonic crystals. In particular, we evaluated several papers that include different approaches such as self-collimation effects, multi-mode interference, waveguide intersection path and nonlinear effects. We then exploited the advantages and weakness of each method. With the resulting information, we addressed the main challenges and possible applications for each scheme. With this review we expect to improve the development of $\mathrm{PhC}$ integrated logic devices and circuits.
\end{abstract}

Index Terms - Photonic Crystal, Logic Gates, Integrated Photonics Technology, Photonic Circuits and Systems.

\section{INTRODUCTION}

It is well known that logic gates are in the third abstraction level of computer logic systems and play a key role to the development of communication and computer systems. Nowadays, they are built connecting transistors based on CMOS technology. Digital electronics is reaching its physical limit of miniaturization, and the incoming future computers with high speed and low power consumption may not be possible with this technology. Thus, it is important to find alternatives to handle this drawback. One possibility is to change to photonics instead of electronics.

Photonic Crystals $(\mathrm{PhC})$ emerged as good candidates to realize fully optical processing information and computing [1-3]. Their ability to control electromagnetic waves, compactness, low power consumption, and exceptional confinement of light, has opened a large variety of applications for telecommunications, signal processing, biophotonics, and computing [4-8].

$\mathrm{PhC}$ logic gates have been explored during the last decade. In this way, several approaches have been applied to build logic devices such as: self-collimation, multi-mode interference, waveguide interference path, semiconductor optical amplifiers, and nonlinear effects. Each technique presents advantages and disadvantages that make them suitable for particular applications.

Thus, this literature review pretends to explore the most common approaches to build photonic crystal logic gates, as well as address possible applications and exposes the main challenges of these technology. This manuscript is organized as follows: Section II gives the fundamental theory to better understand this review. In section III, we make a survey on the most common techniques used to design $\mathrm{PhC}$ logic devices. In section IV, we summarize and compile the review and discuss the main features of each approach. Finally, Section $\mathrm{V}$ gathers the main conclusions.

\section{FUnDAMENTAL THEORY}

This section gives a theoretical review of what photonic crystals are and their fundamental properties. It also covers the kinds of defects that can be introduced in them, the interference and nonlinear effects, and the simulation methods.

\section{A. Photonic Crystals}

Photonic crystals $(\mathrm{PhC})$ are devices that have awaken a great interest since their proposal in 1987, and even more after their experimental demonstration in 1991 [1-3]. Fundamentally, $\mathrm{PhC}$ are a class of optical structures formed by a periodic modulation of the dielectric function distributed in a specific geometric lattice.

Due to this periodicity, electromagnetic waves are affected by the coherent scattering phenomena when they are launched into the $\mathrm{PhC}$. The scattered waves cause the propagation or reflection of particular frequencies inside the structure, as a result of the constructive and destructive interferences between them. The allowed and forbidden frequencies are associated to the photonic bands (PB) and photonic band gaps (PBG), respectively. Thus, when the electromagnetic wave incident on the crystal has frequency within the PBG, it is completely reflected. Otherwise, if the frequency is in the allowed region (PB), it will propagate inside the PhC [9].

As can be noted, this fact means that $\mathrm{PhC}$ operate as optical semiconductor devices and allow us to design insulators or conductors of electromagnetic waves by engineering their geometrical parameters. Photonic crystals can be divided geometrically into three broad categories, namely one-dimensional (1D), two-dimensional (2D), and threedimensional (3D) structures.

In $1 \mathrm{D} \mathrm{PhCs}$, the periodic modulation of the dielectric function occurs in one direction, while in the other two directions the medium is uniform. A very low number of possible variations can be accomplished, in this case: only the dielectric materials, layer's thicknesses, and the number of layers within the period can be varied $[9,10]$.

In a $2 \mathrm{D}$ photonic crystal structure, the periodicity of the dielectric function occurs in two directions, while in the third the medium is uniform. Consequently, a large variety of configurations can be achieved by varying the dielectric materials, lattice geometry (square, hexagonal), lattice constant $a$, and shape of the dielectrics (square, ellipses, triangle). Nevertheless, for technological reason two types of configurations are commonly used: square and hexagonal lattices, illustrated in Figure 1. For both cases, good examples are a silicon substrate with etched holes or a system of dielectric rods in air [9].

The three-dimensional photonic crystals have dielectric modulations along all three directions. With that, the number 


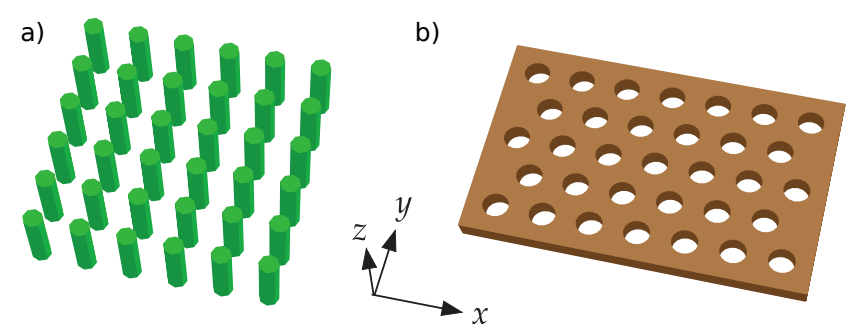

Fig. 1: 2D photonic crystals lattice configurations. a) A system of dielectric rods in air and b) a dielectric substrate with etched holes. Taken from [10].

of possible configurations is much larger than in case of $1 \mathrm{D}$ or $2 \mathrm{D}$ PhC. However, these 3D structures are more difficult to fabricate, although they are relatively common in nature. The most known naturally formed $3 \mathrm{D} \mathrm{PhC}$ is the valuable stone opal [9].

At this point, as in solid state physics, the concept of band diagrams (also called band structure or dispersion relation) is useful to understand the properties of the structure. A band diagram is a plot of the allowed frequencies as a function of the wave vector, usually along a highly symmetric direction in the reciprocal space (the first Brillouin Zone). Its physical meaning is the connection between the characteristics of the photonic crystal and its properties of propagation (PB) and reflection $(\mathrm{PBG})[9,10]$. It can be obtained solving the eigenvalue problem of the master Maxwell equation:

$$
\hat{\Theta} H(r)=\left(\frac{\omega}{c}\right)^{2} H(r)
$$

where $H(r)$ is the magnetic field, $r$ is the (cartesian) position vector, $\omega$ is a given frequency, $c$ the speed of light, and $\hat{\Theta}$ is the hermitian Maxwell operator, defined as:

$$
\hat{\Theta}=\nabla \times\left(\frac{1}{\varepsilon(r)} \nabla \times H(r)\right) .
$$

Here, $\varepsilon(r)$ is the dielectric function and it is the square of the refractive index $n$. In general $\left(n=\sqrt{\varepsilon \mu / \varepsilon_{0} \mu_{0}}\right)$. However, for most dielectric materials of interest, the relative magnetic permeability $\left(\mu(r)=\mu / \mu_{0}\right)$ is very close to unity making $n$ approximately $\sqrt{\varepsilon(r)}$ [10].

In summary, photonic crystals are promising devices considering their properties, which allow the manipulation of photons, like semiconductors allow the control of electrons. Indeed, scientists are now turning to light instead of electrons as the information carriers and have begun imagining photonic integrated circuits which resemble microscopic networks at micrometer length scales. Light has several advantages over the electron, such as it can travel in a dielectric material at much greater speeds, can also carry a larger amount of information per second, the bandwidth of dielectric materials is significantly larger than that of metals, and light particles (or photons) are not as strongly interacting as electrons, which helps reduce energy losses [11].

A..1 Photonic Crystal SlabControl the light in all three dimensions is the goal of the technological and scientific community. To do that, three-dimensional photonic crystals are the ideal platform to confine and guide the light in the three dimensions. However, 3D PhC are particularly difficult to realize because large-area and defect-free implementations are quite challenging even for state-of-art fabrication techniques [12-14]. Two-dimensional photonic crystals embedded into planar dielectric waveguides, known as two-dimensional $\mathrm{PhC}$ slabs, have emerged in the last decade as the best candidates for efficiently confining and guiding the electromagnetic fields at optical frequencies in three dimensions. Currently, lithography and etching processes at sub-micron scales are in a very mature and advanced stage, allowing the fabrication of large-area photonic crystal slabs with high-nanometric precision [15].

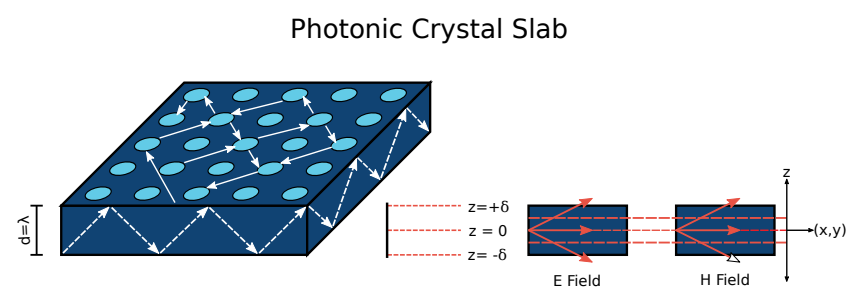

Fig. 2: Schematic representation of a photonic crystal slab. The dashed lines indicates the TIR mechanism along the $z$ axis. The solid line represent the DBR mechanism on the 2D pattern. The electromagnetic modes on a $\mathrm{PhC}$ slab are confined at $z=0$. Generally, the thickness of the slab is $d \sim \lambda$, where $\lambda$ is the operating wavelength of the device.

In $\mathrm{PhC}$ slabs, the vertical confinement mechanism is given by total internal reflection (TIR), and in-plane the propagation is controlled by the photonic pattern via distributed Bragg reflection (DBR), as shown in Figure 2. Photonic band gaps in these systems are thus conditioned by both TIR and DBR mechanisms.

The electromagnetic modes in a PhC slab can be divided into two orthogonal polarizations, known as transverseelectric (TE) and transverse-magnetic (TM). The first has field components $\left(E_{x}, E_{y}, H_{z}\right)$, while the second presents the following components $\left(H_{x}, H_{y}, E_{z}\right)$, considering $x y$ as the plane where the photonic pattern is present.

\section{B. Photonic Crystal Defects}

In particular, two types of defects can be introduced in two-dimensional photonic crystals: point defects and line defects, known as cavities and waveguides, respectively.

B..1 Photonic Crystal CavityA photonic crystal cavity is a perturbation of the system that breaks the periodicity of its dielectric function. This can be made removing one or more dielectric features, a dielectric rod or a hole in the structure, or replacing them with other features, whose size, shape or dielectric constant are different from the original.

In effect, if the designed defect has the proper size, shape and dielectric constant to support an electromagnetic mode within the photonic band gap, the light cannot escape from it, and it is trapped into the cavity forming an optical resonator, as illustrated in Figure 3. The two most important characteristics of interest in $\mathrm{PhC}$ cavities are: the mode volume and the quality factor $(Q)$.

The mode volume is a measure of how tightly the mode is localized into the cavity. It can be determined with the dielectric function and the field function of the electromagnetic mode profile [16]. 
The quality factor $Q$ is a dimensionless quantity that relates the outgoing power and the electromagnetic energy localized in the cavity. Also, it can be viewed as a measure of the number of optical periods that elapse before the energy decays, i.e., the lifetime of the electromagnetic modes in the cavity [10]. The quality factor of a $\mathrm{PhC}$ cavity can be computed using the following relation:

$$
\frac{1}{Q}=\frac{P}{\omega_{0} U},
$$

where $P$ is the outgoing power, $\omega_{0}$ is the resonant frequency and $U$ is the electromagnetic energy localized in the cavity [10]. A high $Q$ implies a longer lifetime of the electromagnetic waves inside the defect.

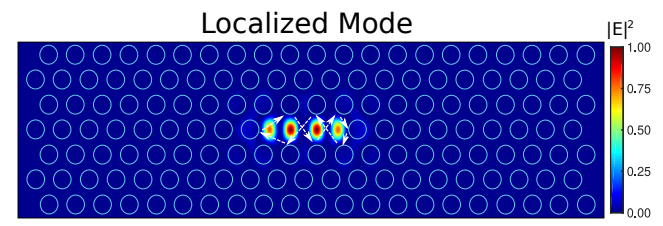

Fig. 3: Photonic crystal cavity. Schematic representation and normalized intensity distribution of localized mode in a photonic crystal L3 cavity.

High quality factors have been achieved in twodimensional photonic crystals using the L3 cavity. It is created by removing three holes or rods in the same line of the structure, as shown in Figure 3. Values of $Q$ reaching the order of $10^{5}$ have been reported in the literature for $\mathrm{L} 3$ cavities [17].

Such kind of cavity has important applications for low threshold lasers, high finesse filters, sensors, cavity quantum electrodynamics as well as filtering, switching, and integrated optical processing when integrated with waveguides [18-22].

B..2 Photonic Crystal WaveguideAnother fundamental type of perturbation in a photonic crystal structure is a waveguide. Essentially, a waveguide in a two-dimensional photonic crystal device is a line defect created by removing or changing the properties of one entire dielectric row or column, as shown in Figure 4.

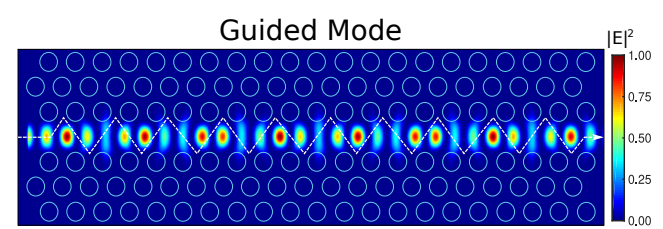

Fig. 4: Photonic crystal waveguide. Schematic representation and normalized intensity distribution of guided modes in a photonic crystal waveguide.

The key difference between cavities and waveguides is that, for the former electromagnetic modes can be localized whenever its frequency is in the photonic band gap, while for the latter the behavior of the mode relies on its frequency and wave vector. Thus, a guided mode needs only the combination of wave vector and frequency disallowed in the structure; it is not necessary that frequency alone is disallowed. With this condition, the conservation of the wave vector along the defect allows the propagation of the electromagnetic mode from one side to another, as opposed to being just a region where it is localized [10].

Taking advantage of this, waveguides are used to guide the electromagnetic waves with very high efficiency and optical devices working in the low losses and low energyconsumption regime for telecommunication purposes can be achieved.

B..3 Photonic Crystal Coupled SytemsA coupled system is one in which the operation of each component is described according to the behavior of the other elements that compose the system.

Waveguides and cavities can form a coupled system in a photonic crystal in order to build efficient frequency filter devices. There are two possible configurations to couple waveguide and cavities in a $\mathrm{PhC}$ structure: side-coupled and directly-coupled.

In a waveguide-cavity directly coupled system, the cavity is placed in line with the waveguide, as illustrated in Figure 5. Thus, light from the input waveguide couples into the cavity, and the cavity in turn couples into the output waveguide allowing the transmission of frequencies near to the resonant frequency of the cavity. This system acts as a narrow-band filter device [10,23].

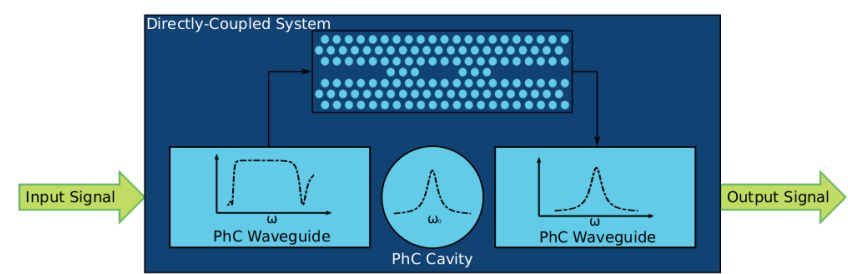

Fig. 5: Representation of a directly waveguide-cavity coupled system in $\mathrm{PhC}$.

The transmission spectrum $(T)$ of this system can be estimated using the following function:

$$
T(\omega)=\frac{\delta}{\left(\frac{\omega-\omega_{0}}{\omega_{0}}\right)^{2}+\delta},
$$

where $\omega_{0}$ is the resonant frequency of the cavity and $\delta$ is a quantity related to the quality factor as: $1 / Q^{2}$.

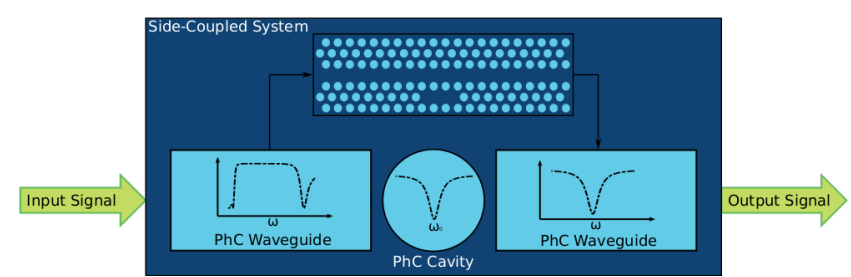

Fig. 6: Representation of a side waveguide-cavity coupled system in $\mathrm{PhC}$.

On the other hand, in a waveguide-cavity side coupled system, shown in Figure 6, the cavity is placed in a line up or down to the waveguide. Thus, the cavity couples with the input and output waveguides reflecting the frequencies around the resonant frequency of the cavity $[10,23]$. For this kind of configuration, the transmission spectrum can be computed 
with the equation:

$$
T(\omega)=\frac{\left(\frac{\omega-\omega_{0}}{\omega_{0}}\right)^{2}}{\left(\frac{\omega-\omega_{0}}{\omega_{0}}\right)^{2}+\delta}
$$

These coupled systems have been used successfully to enhance nonlinear effects, frequency filters, absorptive devices and sensors [24].

\section{Interference Effect Based on MMI}

Generally, Multi-Mode Interference (MMI) devices are composed of the access waveguides, the multi-mode region, and the output waveguides. Its operation principle is the selfimaging property. In this, guided modes are excited and interfere constructively or destructively at the MMI region when an input field is launched at the access waveguides. Then, the excited field at the MMI region transmutes periodically along the propagation direction to the output waveguides. Usually, access and output waveguides are singlemode to ensure a high-performance MMI device. On the other hand, the MMI region is a waveguide that supports a large number of modes and where the MMI occurs [25].

\section{Interference Effect Based on Self-Collimation}

Self-Collimation (SC) or self-guiding of light in a $\mathrm{PhC}$ is a process in which a narrow beam of electromagnetic wave can propagate without any significant broadening or change in the beam profile, and without relying on a bandgap or engineered defects, such as waveguides. This phenomena occurs due to the complex spatial dispersion properties of planar photonic crystal generating anomalous refraction of light. To achieve the $\mathrm{SC}$ in a $\mathrm{PhC}$, the allowed wavevectors in the structure and their corresponding frequencies must be as flat as possible to ensure the perpendicularity of the group velocity of light. Thus, launching beams signals with appropriate phases into a $\mathrm{SC} \mathrm{PhC,} \mathrm{the} \mathrm{reflected} \mathrm{and} \mathrm{transmitted}$ can generate constructive or destructive interference [26].

\section{E. Interference Effect Based on Waveguide Intersection Paths}

As described earlier, $\mathrm{PhC}$ waveguides are used to guide electromagnetic modes from one side to another. $\mathrm{PhC}$ bend waveguides based on square and triangular lattices have been extensively studied and also experimentally demonstrated. Two rules of thumbs can be applied to adequately design the intersection path between two $\mathrm{PhC}$ waveguides. In general, a path difference of zero implies that constructive interference occurs between the two input signals. In contrast, a destructive interference will occur if the path difference between the waveguides are one lattice constant $[27,28]$.

\section{F. Kerr Effect in Photonic Crystals}

The optical Kerr effect is a kind of phenomena in that a nonlinear material changes its refractive index under high intensity radiation passing over them. This change of the refractive index can be calculated through the following equation:

$$
\Delta n=n_{0}+n_{2} I
$$

where $n_{0}$ is the linear refractive index, $n_{2}$ is the Kerr coefficient and $I$ is the intensity of the electromagnetic wave. Such variations of the refractive index can cause essential changes of device fundamental characteristics [29].

In this way, the best approach to observe the optical Kerr effect in photonic crystals is through a coupled system with a nonlinear cavity. In this kind of system, the introduced nonlinearity induce a change of the refractive index inside the cavity which follows the relation of the Equation 6 . Besides, it is observed that the cavity resonant frequency shifts proportional to $\Delta n$ due to the subsequent growth of the electromagnetic energy inside the resonator [24].

Then, after applying a rigorous perturbation theory arguments, the relation between the output and input power in a directly-coupled system can be expressed by the Lorentzian [24]:

$$
\frac{P_{\text {out }}}{P_{\text {in }}}=\frac{1}{1+\left(P_{\text {out }} / P_{0}-\Delta\right)^{2}}
$$

where $P_{\text {in }}$ and $P_{\text {out }}$ are the input and output powers, $P_{0}$ is the characteristic power of the cavity, and $\Delta$ relates the resonant frequency $\left(\omega_{0}\right)$ with the width of the resonant $(\Gamma)$, $\Delta=\omega-\omega_{0} / \Gamma$. For a side waveguide-cavity coupled system, this relation can be expressed as:

$$
\frac{P_{\text {out }}}{P_{\text {in }}}=\frac{\left(P_{\text {out }} / P_{0}-\Delta\right)^{2}}{1+\left(P_{\text {out }} / P_{0}-\Delta\right)^{2}}
$$

In general, if the applied input on the waveguide has the proper amount of intensity to put in the cavity on the nonlinear regime, then the resonant frequency of the system shifts due to the change in the refractive index of the nonlinear cavity. This variation gives the possibility to design a new class of optical devices such as optical information storage elements, bistables, transistors, and logical elements [22, 30-37].

\section{G. Simulation Methods}

G..1 Finite Difference Time Domain MethodThe finite difference time domain method (FDTD) is a numerical technique used to solve Maxwell's equations. It can be implemented without using linear algebra which makes it appropriate for rigorous simulations of large scale problems, and structures with non-uniform dielectric distribution and complicated geometries. It is accurate, and sources of numerical error are well understood. Also, FDTD calculates naturally the nonlinear response of an electromagnetic system as it is a time-domain technique [38,39].

To solve Maxwells equations, FDTD divides space and time into a grid (usually uniform) of discrete points and approximates their derivatives $(\nabla \times$ and $\partial / \partial t)$ by finite differences. The propagation in time, in particular, uses a leap-frog scheme where the $E$ fields at time $t$ are computed from the $E$ fields at time $t-\Delta t$ along with the $H$ fields at time $t-\Delta t / 2$, and vice versa for $H$ at $t+\Delta t / 2$. In this way, the $E$ and $H$ field patterns are marched through time, offset by half of a time step $\Delta t$ [38-40].

Nowadays, FDTD is one of the most advanced methods for computation the field distribution and to study other behaviors such as nonlinearities in photonic crystals. It had 
also been applied to a large variety of electromagnetic problems $[38,39]$. There are some commercial and open source software packages that implements the FDTD method, such as MEEP, OptiFDTD, Lumerical FDTD Solution Tool and RSoft.

G..2 Plane Wave Expansion MethodThe Plane Wave Expansion (PWE) method is a frequency-domain eigensolver which solves the eigenproblem for the frequencies of a periodic system such as photonic crystals.

The discretization of the master Equation 1 through planewave expansion yields a finite generalized eigenproblem $A x=\omega^{2} B x$, where $A$ and $B$ are $N \times N$ matrices, $x$ the eigenvector and $\omega$ the eigenvalues. Here, the eigenvalues represent the frequencies while the eigenvectors contain amplitude coefficients of the component plane waves [41-43]. In particular, for a one dimensional $\mathrm{PhC}$ structure, the plane wave using Fourier series takes the form:

$$
u_{k}(x)=\sum_{n=-\infty}^{\infty} c_{n}(k) e^{i \frac{2 \pi n}{a} x},
$$

where $u_{k}(x)$ is a periodic function $u_{k}(x)=u_{k}(x+a)$, where $a$ is the lattice constant. $c_{n}(k)=\frac{1}{a} \int_{0}^{a} d x e^{-i \frac{2 \pi n}{a} x} u_{k}(x)$ are the complex Fourier series coefficients.

In general, PWE method may be the most popular approach for calculating band diagrams of periodic structures like photonic crystals. It is a simple method to implement and enables rigorous analysis of 3D structures [10]. A free software tool called MPB (MIT Photonic Band-gap) is available for this purpose.

\section{Photonic Crystal Logic Gates}

This section presents a literature review of several methods used to perform logic devices in photonic crystals.

\section{A. LG Based on Self-Collimation (SC)}

An incident light beam is partially reflected and partially transmitted when incident a PhC structure with a selfcollimated region. Thus, a phase change occurs in the reflected beam with respect to the transmitted beam. This phase difference dependents on the radius of the dielectrics of SC PhC region. If another light beam with appropriate phase is launched, the reflected and transmitted beams may interfere constructively or destructively. Thus, logic gates based on SC PhC can be achieve by modifying the radii of the dielectrics and introducing different phase shifts between the incident beams at the input faces. Several works using this approach demonstrated a complete set of all-optical logic gates [44-47]. Figure 7 shows the schematic structure of the photonic crystal self-collimated device proposed by Zhang et al [44]. This device is formed by two input faces $\left(I_{1}, I_{2}\right)$ and two output faces $\left(O_{1}, O_{2}\right)$. It can operate as OR and XOR logic gates by introducing a certain phase difference between the two beams (incident on the input faces $I_{1}$ and $I_{2}$ ). Thus, if the phase difference between the inputs $\phi_{1}\left(I_{1}\right)-\phi_{2}\left(I_{2}\right)$ is sets as $2 k \pi+\pi / 2$, the output face $O_{1}$ and $\mathrm{O}_{2}$ operates as OR and XOR logic gates, respectively.

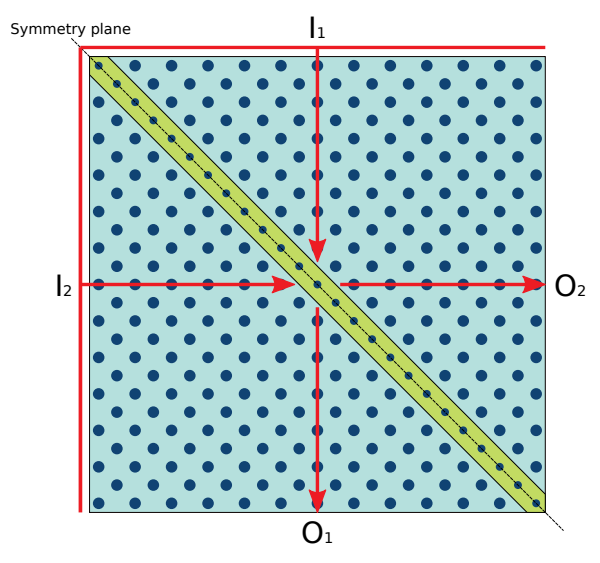

Fig. 7: Schematic representation of photonic crystal logic device based on self-collimated effect proposed by Zhang et al [44]. Adapted from [44].

\section{B. LG Based on MMI (Multi-Mode Interference)}

To accomplish logic gates based on MMI, the input logic values need to be determined only by the phase of the input signals for each gate. This can be achieved by using Binary Phase Shift Keyed (BPSK) signals, whereas the output logic value is determined by the amplitude of the output signal regardless of its phase. With the proper signal phases of the input signals, they interact together in the MMI area to either generate a signal, which corresponds to logic 1 , or eliminate the signal generation, which corresponds to logic 0 at the output port. Then, the logic gates can be realized by the proper parameters selection in addition to the choice of the phases of the input signals, as demonstrated by Ishizaka et al., Liu et al., and Tang et al. [48-51].

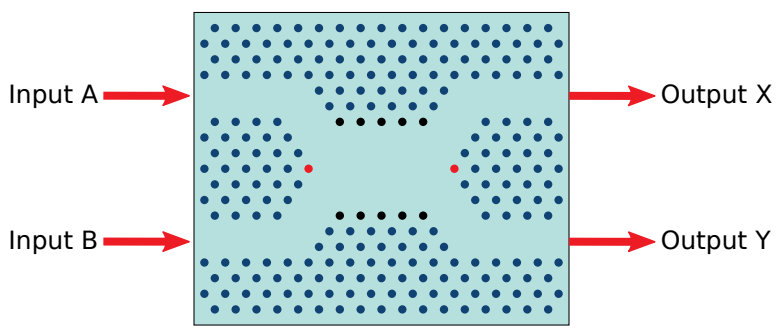

Fig. 8: Schematic representation of photonic crystal logic device based on multi-mode interference proposed by Liu et al [49]. Red and black circles represent optimized rods. Adapted from [49].

Figure 8 shows the schematic representation of the $\mathrm{PhC}$ MMI device proposed by Liu et al [49]. It is formed by two input ports (A and B) and two output ports (X and Y). Thus, to achieve the logical functions, two kinds of BPSK signals are injected into each input port. In the case of XNOR gate, logic 1 for the input port $\mathrm{A}$ is expressed as signal phase 0 , while logic 0 is expressed as signal phase $\pi$. Whereas the input port $\mathrm{B}$ expresses logic 1 as signal phase $-\pi / 2$, while logic 0 is expressed as signal phase $\pi / 2$. Similarly, the XOR, OR and NAND gates can be realized by selecting the proper input signal's phase for each logic. 


\section{LG Based on Waveguide Intereference Paths}

This is a simple, useful, and effective approach to project logic gates in photonic crystals. In this scheme, the high transmission state (logic 1) is guaranteed if a constructive interference occurs between the inputs waveguides. This is accomplished by designing an intersection point with a path difference of 0 , which generate a phase difference of $2 k \pi$ between input signals. In contrast, if the designed path difference is one lattice constant, it generates a phase difference of $(2 k+1) \pi$, and destructive interference occurs between the inputs signals achieving the low transmission state (logic 0 ).

Using these approach, the OR, XOR, NOT, XNOR, and NAND gates have been realized theoretically by $\mathrm{Fu}$ et al. [27]. The structures were projected on a photonic crystal composed of a triangular lattice of cylindrical silicon rods, with a dielectric constant of 11.56 , embedded in a background medium of air (refractive index 1). The lattice constant and the diameter of the silicon rods were $875 \mathrm{~nm}$ and $495 \mathrm{~nm}$, respectively. The OR logic gate is formed by two intersecting waveguides (inputs) at $10.5 \times 10^{3} \mathrm{~nm}$ to the cross point between them, forming an angle of 120 and a phase difference of 0 , as illustrated in Figure 9a. Thus, if a single beam is injected into one of the inputs, the signal light can propagate to the output through the waveguide, and a logical value of 1 can be obtained in the output. When two beams are injected in both input ports simultaneously, constructive interference occurs, and high power is observed in the output. Obviously, when no single beam is injected into any input port, no light comes to the output, corresponding to the logical value of zero.

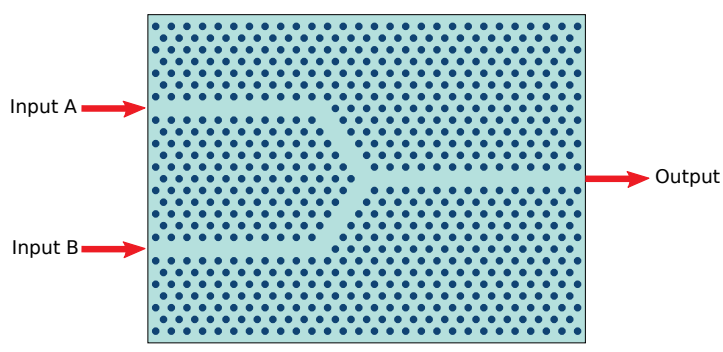

(a)

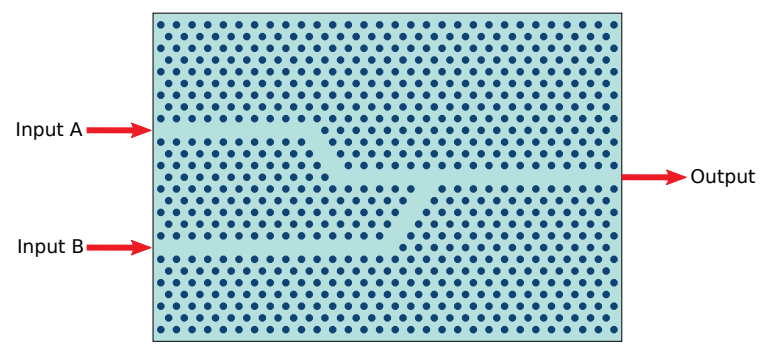

(b)

Fig. 9: Schematic representation of photonic crystal (a) OR and (b) XOR gates proposed by Fu et al. [27]. Adapted from [27].

For the XOR gate, the designed structure consists of two waveguides with one lattice constant of path difference to the cross point between them, as shown in Figure 9b. At this point, when the inputs are excited simultaneously with a continuous wave source, a phase difference of $\pi$ generates a destructive interference, and the output signal is approximately zero $(0.67 \%)$. On the other hand, if only one input is excited, the output signal is greater than $75 \%$. Thus, considering transmissions greater than $70 \%$ and lower than $1 \%$ as logic values 1 and 0 , respectively, the XOR logic gate was carried out.

The XNOR and NAND gates structures were based on the XOR device, but introducing a control signal in order to generate a high transmission output when no light is injected into the input ports, i.e for the logic input case $(0,0)=1$. Thus, for these devices, transmission values greater than $85 \%$ and lower than $10 \%$ have been reported for logic values 1 and 0 , respectively. The logic devices proposed can operate at the telecommunication window of $1550 \mathrm{~nm}$ and in the low power regime. Also, the author reported the intensity contrast ratio between the output signals for the logic states of 1 and 0 as high as $20 \mathrm{~dB}$.

Using the same parameters, Caballero et al. addressed the Majority and the Feynman gates [52]. The former allows the creation of simple and optimized computational circuits and the latter is a reversible logic device projected to design circuits in the thermodynamic limit of computation. For the Majority gate, illustrated in Figure 10a, the authors reported that transmission values at the output greater than $85 \%$ and lower than $35 \%$ can be interpreted as logic 1 and 0 , respectively. For the Feynman gate, shown in Figure 10b, the equivalent transmission for the logic 1 was found as high as $40 \%$ while transmissions values lower than $10 \%$ correspond to the logic 0 .

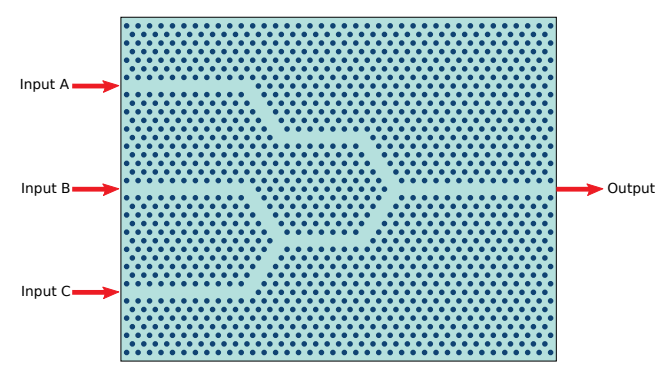

(a)

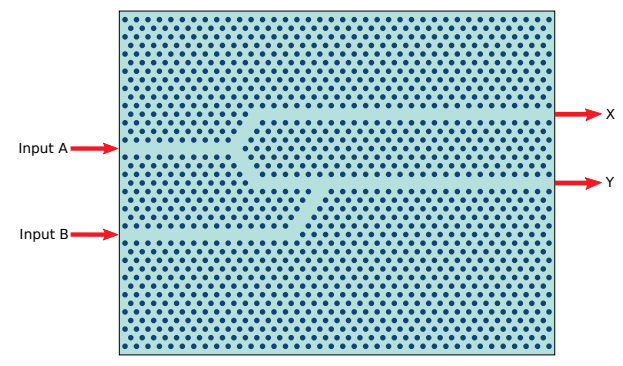

(b)

Fig. 10: Schematic representation of photonic crystal (a) Majority and (b) Feynman gates proposed by Caballero et al. [52]. Adapted from [52].

Employing the control interference effect in a twodimensional photonic crystal composed of a square lattice of cylindrical silicon rods, with a dielectric constant of 11.56 , embedded in a background medium of air, D'Souza demonstrated the operation of the OR, XOR, NOT, and AND logic 
gates [28]. The parameters of the based structure were 650 $\mathrm{nm}$ for the lattice constant and $230 \mathrm{~nm}$ for the radii of the rods. The devices are formed by a square ring resonator waveguide with three other linear waveguides which are connected to each other by the ring resonator, as illustrated in Figure 11. The principle is that the signal injected into the input waveguides split into two through the ring resonator. One travels in clockwise and the other in the counterclockwise direction. If a constructive interference occurs, a larger output energy is obtained at the output and the OR, and AND gates were accomplished. Engineering the ring resonator to produce destructive interference, the XOR and NOT logic gates were also demonstrated. The advantage of these devices is that they can operate at different wavelengths around the $1550 \mathrm{~nm}$ window. In addition, a contrast ratio higher than $35 \mathrm{~dB}$ was reported by the authors.

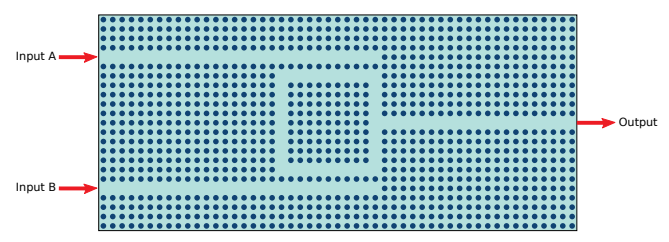

(a)

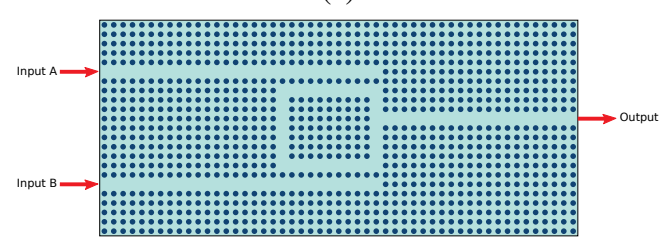

(b)

Fig. 11: Schematic representation of photonic crystal (a) AND and (b) XOR gates proposed by D'Souza et al.[28]. Adapted from [28].

A methodology to evaluate the effect of structural disorder on photonic crystals logic gates were applied to these devices in order to figure out their robustness and fault tolerance [53]. The study was based on the evaluation of two metrics: the error rate (ER) and the mean absolute deviation of transmission of the error cases (MATEC). ER is the probability that a fabricated photonic crystal logic gate does not accomplish its logic function correctly, and MATEC measures the imperfection degree of the device through the transmission coefficient. The authors found that, for structures with a triangular lattice, regions in the corners and close to the output are more critical to ER and MATEC, respectively. For structures with a square lattice, the intersection regions are the most sensitive for both metrics.

Hussein et al. introduce new designs of all-optical OR, AND, XOR, NOT, NOR, NAND and XNOR logic gates based on the interference effect [54]. The designs were projected using 2D square lattice photonic crystal structures of germanium $(\mathrm{Ge})$ rods, with a relative permittivity of 16 , embedded in a background of air. The radius of the rods was set as $0.15 a$, where $a$ is the lattice constant and its value was selected $580 \mathrm{~nm}$ to get a operational wavelength of $1550 \mathrm{~nm}$. The interference effect to achieve the logical function is projected via an optical resonator induced for each structure, as shown in Figure 12. Optical bit rates ranging from 3.8 to
7.6 Tbps and contrast ratio from $5.036 \mathrm{~dB}$ to $12.15 \mathrm{~dB}$ were reported in this work.

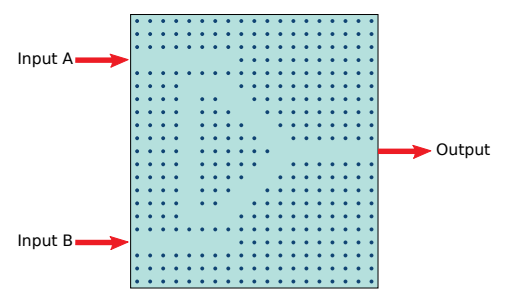

(a)

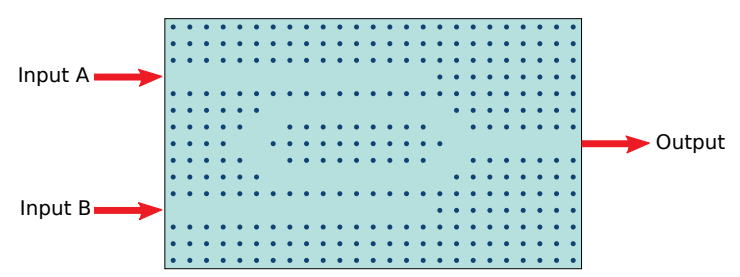

(b)

Fig. 12: Schematic representation of photonic crystal (a) AND and (b) OR gates proposed by Hussein et al. [54]. Adapted from [54].

Caballero et al. [55] accomplished an integrated and compact photonic crystal $(\mathrm{PhC})$ device that can operate as a NAND or NOR logic gate, shown in Figure 13. It is designed in a $\mathrm{PhC}$ slab composed of GaAs/AlGaAs heterostructure and a $2 \mathrm{D}$ pattern of a triangular lattice of holes. By modifying target holes on the structure, the NAND or NOR gates can be achieved. The simulation results reported by authors show that the upper power limit to represent the logic 0 is 0.17 Pin, where Pin is the input power. On the other hand, the lower power limit to represent the logic 1 is found to be $0.50 P i n$. The NAND and NOR logic gates present a response time of $5 \mathrm{ps}$, resulting in a clock rate of $200 \mathrm{GHz}$. They also operate within the $\mathrm{C}$ band of the telecommunication window (between 1530 and $1565 \mathrm{~nm}$ ).

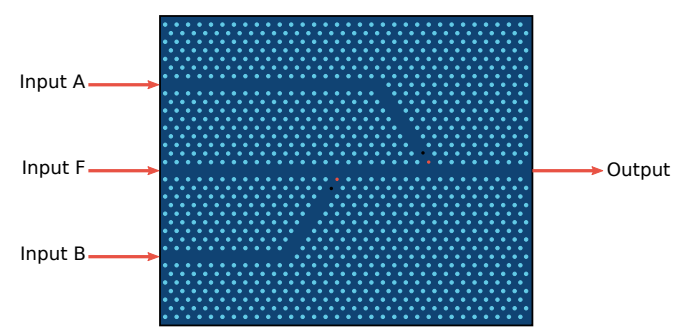

Fig. 13: Schematic representation of the XY view of the integrated photonic crystal compact device. In this, dark and light colors correspond to two different dielectric constant $\varepsilon_{1}$ and $\varepsilon_{2}$, respectively. With $\varepsilon_{1}>\varepsilon_{2}$. Red and black circles represents the target holes to be optimized to achieve the NAND and NOR logic functions in our PhC structure. Adapted from [55].

\section{LG Based on Kerr Effect}

To accomplish logic devices using the Kerr effect on $\mathrm{PhC}$, a waveguide-cavity coupled system need to be introduced on the structure and the power of the inputs need to be properly selected. 
Using this scheme, a high-contrast all-optical bistable switching was proposed by Yanik et al. [22]. The projected device is a $2 \mathrm{D}$ photonic crystal composed of a square lattice of dielectric rods $(\varepsilon=12.25)$ embedded in air, as illustrated in Figure 14. To achieve the optical bistability, a waveguide was side-coupled to a single-mode cavity with instantaneous Kerr nonlinearity. Consequently, the high transmission state is accomplished when the cavity is OFF resonance with the excitation and the field inside the cavity is low, making the decaying field amplitude from the cavity negligible. In contrast, when the field intensity inside the cavity is much higher, it pulls the cavity resonance frequency down to the excitation frequency of the incident field, and the low transmission state is achieved. To prove the correctness of the bistability, a coupled-mode theory was applied and presented excellent agreement with FDTD simulations.

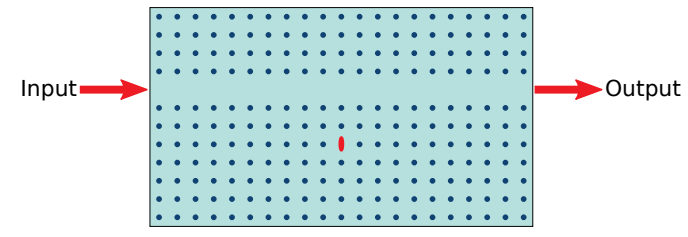

Fig. 14: Schematic representation of the photonic crystal Switch proposed by Yanik et al [22]. Red ellipses represent nonlinear materials. Adapted from [22].

In a following work performed by the same research group, the first all-optical transistor in photonic crystals was proposed [31]. The structure consists of two waveguides, input and control, arranged in a cross geometry. Additionally, a cavity with instantaneous Kerr nonlinearity was inserted in the waveguides intersection, as shown in Figure 15. With this configuration, the cross-talk between the waveguides is prohibited due to the modes of them are even to their axis; consequently, each waveguide couples to only the cavity mode with the same axis symmetry. FDTD simulations were performed to show the transistor operation. Thus, the ON state is reached at $25 \mathrm{ps}$ when the input and control waveguides are excited with input powers about $200 \mathrm{~mW} / \mu \mathrm{m}$. Otherwise, if only the input waveguide is launched, the transistor is on the OFF state. A small footprint of a few micrometers squared and the requirement of only a few milliwatts of power at a $10 \mathrm{Gbit} / \mathrm{s}$ switching rate were considered the advantages of the proposed structure.

In the search for useful multipurpose devices, an alloptical half adder based on photonic crystals resonant cavities was proposed by Neysi et al. [56]. The designed structure is composed of a $31 \times 31$ square array of dielectric rods $(\varepsilon=11.97)$ immersed in air. Two inputs waveguides and two nonlinear resonant cavities, as can be observed in Figure 16 , were introduced and optimized to get the correct behavior for the resonant frequencies. To prove the functionality as a half adder, a continuous wave with a wavelength of 1551 $\mathrm{nm}$ and an optical intensity of $10 \mathrm{~mW} / \mu \mathrm{m}^{2}$ is used as input source. Values greater than $70 \%$ and lower than $5 \%$ were reported to be considered as logic 1 and logic 0 , respectively. Compactness and low delay rate of about 3 ps are highlighted as the main advantages of the proposed structure.

In the same direction, a photonic crystal 1-bit full adder

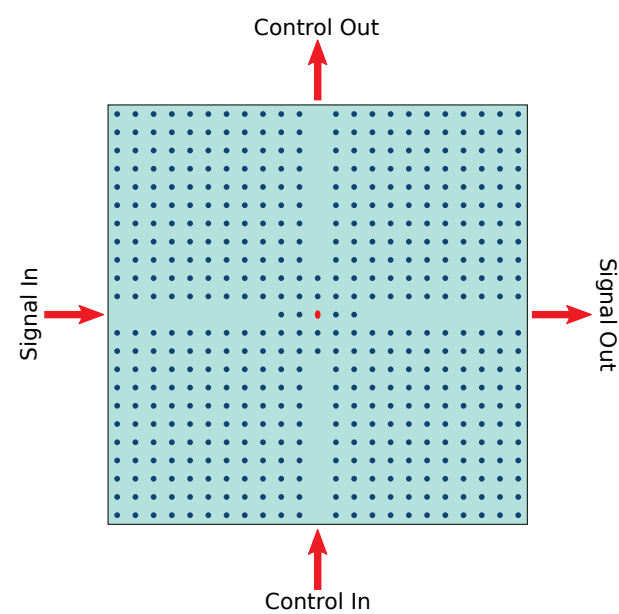

Fig. 15: Schematic representation of the photonic crystal Transistor proposed by Yanik et al [31]. Red ellipses represent nonlinear materials. Adapted from [31].

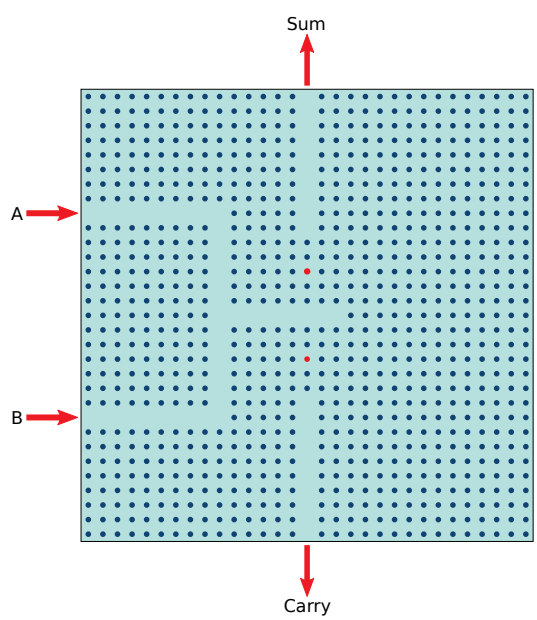

Fig. 16: Schematic representation of the photonic crystal half adder proposed by Neisy et al [56]. Red circles represent nonlinear materials. Adapted from [56].

was projected by Alipur-Banei et al. [57]. The structure was developed by cascading two optical 1-bit half-adders. The final structure consists of eight optical waveguides and two nonlinear resonant rings, created inside rod type twodimensional photonic crystal with square lattice. The structure has $\mathrm{X}, \mathrm{Y}$ and $\mathrm{Z}$ as input waveguides and, SUM and CARRY as output ports waveguides. The performance and functionality of the adder were validated by means of the FDTD method. The results shown that normalized transmission values greater than $60 \%$ and less than $5 \%$ were considered as logic 1 and logic 0 , respectively. In addition, a delay time of $1.5 \mathrm{ps}$ and the overall footprint of about $439 \mu \mathrm{m}^{2}$ were reported.

The energy dissipation produced by the information loss is currently a concern in the design and fabrication of logic circuits. R. Landauer showed that there is a great amount of energy dissipation inside large scale digital circuits because of information loss [59], which is an inevitable phenomenon in irreversible logic gates. However in reversible logic gates due to one by one mapping between the input ports and output ports, it is possible to obtain the state of input ports from 


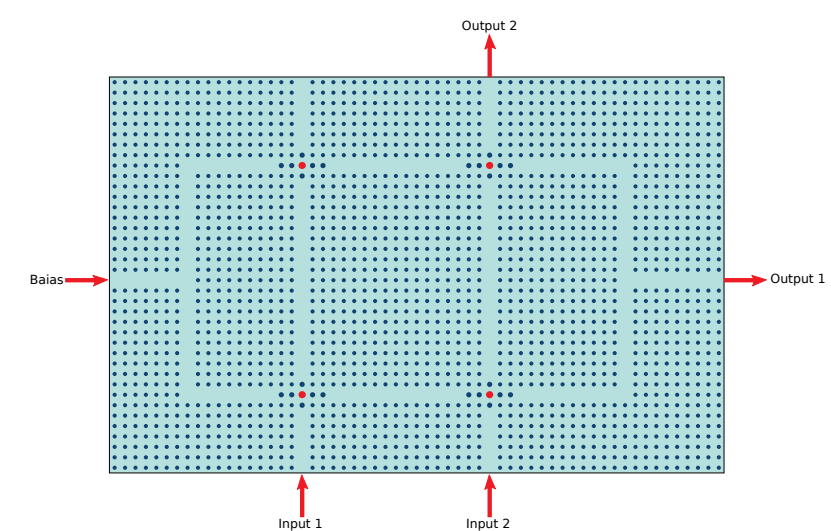

Fig. 17: Schematic representation of the photonic crystal reversible XOR and XNOR gates proposed by Hassangholizadeh-Kashtiban et al [58]. Red circles represent nonlinear materials. Adapted from [58].

the output ports. As a result, no information loss is presented, so the energy dissipation will be reduced. Thus, all-optical reversible XOR and XNOR gates based on electromagnetic scattering phenomenon in nonlinear photonic crystal structures were reported [58]. The projected devices consist of two cross-connected waveguides acting as inputs and outputs along with some linear and nonlinear defect rods, illustrated in Figure 17. A 2DPhC with cubic lattice was used as the fundamental structure. The operation principle to get the XOR reversible logic is to map one of the inputs to one of the outputs, and compute in the second output the conventional XOR operation. For the XNOR, the inverse of one input is mapped to one of the outputs, and the conventional XNOR operation is performed in the second output. The results showed the correct operation of the logic gates for transmission values greater than $65 \%$ and lower than $2 \%$ considered as logic 1 and logic 0 , respectively. The authors also reported a 10 ps of a maximum time delay to get the logic answer.

Recently, Caballero et. al [60] reported the complementary photonic crystal integrated logic devices (CPCL). It consists of two logic devices named as Switch N and P, shown in Figure 18. The former is the equivalent of NMOS logic transistor and can operate as AND gate. The later can work as an inverter and as PMOS logic transistor. Simulation results provided by authors demonstrate a highly efficient clock rate, higher than $20 \mathrm{GHz}$, guaranteeing operation at both input and output with the same wavelength (around $\lambda=$ $1550 \mathrm{~nm}$ ). Also, they found that the proposed devices show well-defined output power values representing the two logic states 1 and 0 , with a contrast ratio as high as $6 \mathrm{~dB}$. This fact allows, for the first time, the development of photonic integrated universal logic circuits, such as CMOS transistors for electronic digital circuits, reaching a high computing abstraction level, switching up from basic components to computational systems.

Some other strategies have been addressed to project PhC logic gates. Examples of these are: semiconductor-opticalamplifier (SOA), PhC fiber, topological cavity and edge states, stimulated Raman scattering, Mach-Zehnder interferometer [61-65]. All of these works reviewed here prove that

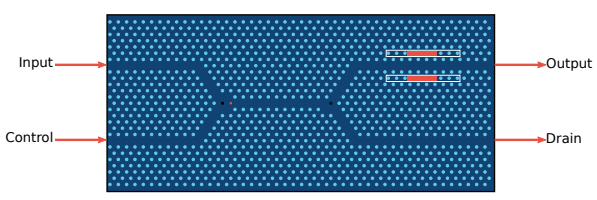

(a)

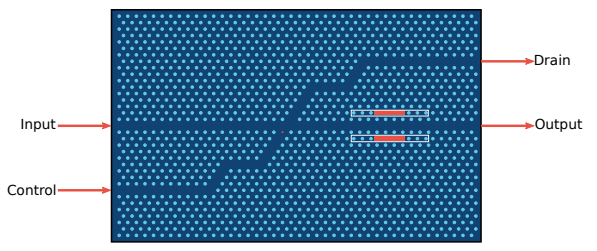

(b)

Fig. 18: Schematic representation of XY view of the photonic crystal integrated switch N (a) and (b) switch P. In this, dark and light colors correspond to two different dielectric constant $\varepsilon_{1}$ and $\varepsilon_{2}$, respectively. With $\varepsilon_{1}>\varepsilon_{2}$. Black and red circles represent optimized holes with radii $0.75 r$ and $0.5 r$, respectively. The displacements of the holes around the cavity are $S 1=0.33 a, S 2=0.26 a, S 3=0.12 a$. Adapted from [60].

$\mathrm{PhC}$ are good candidates to develop computational logic devices with low power consumption, high-efficiency and with high-speed data processing.

\section{Discussion}

In this section we discuss the advantages and disadvantages of the strategies to project $\mathrm{PhC}$ logic gates, together with a description of the most relevant achievements of this review.

$\mathrm{PhC}$ logic gates based on self-collimation present small area, faster response, wide operating frequency bandwidth, and low power operation. Indeed, their size can be reduced to the operating wavelength order. They are also independent of light intensity allowing operation in a low power regime. However, they lack a high contrast ratio, and their microfabrication is challenging to achieve since it is hard to control the dispersion relations to achieve the self-collimation effect. Besides, they need phase shifters to synchronize input signals, increasing the device size and limiting their adjacent connection. LG based on this approach can be suitable for hybrid system integration, signal processing, extreme environments sensors, and frequency shifters.

$\mathrm{PhC}$ logic gates based on MMI exhibit small size, faster response, large operating frequency bandwidth and low power operation. The compactness of the MMI region ensures their simple configuration, robustness and low loss. In addition, low power operation is accomplished because their light intensity independent. Nevertheless, they need to adopt different phase values to achieve the logic state representations. This fact brings inconvenience towards the development of integrated circuits because it increases their size and leads to hard fabrication processes. In general, this scheme can be used to project extreme environments sensors, frequency shifters, splitters, demultiplexers and signal processing.

In general, $\mathrm{PhC}$ logic gates based on waveguide interference paths have wide operating frequency bandwidth, low 


\begin{tabular}{|c|c|c|c|}
\hline Logic Gate Method & Advantages & Weakness & Possible Applications \\
\hline $\begin{array}{l}\text { Self-Collimation } \\
\text { Refs. [44-47] }\end{array}$ & $\begin{array}{l}\text { Small area } \\
\text { Faster response } \\
\text { Wide operating frequency bandwidth } \\
\text { Low power operation }\end{array}$ & $\begin{array}{l}\text { Low contrast ratio } \\
\text { Hard microfabrication } \\
\text { Input phase requirement }\end{array}$ & $\begin{array}{l}\text { Hybrid system integration } \\
\text { Signal processing } \\
\text { Extreme environments sensors } \\
\text { Frequency shifters }\end{array}$ \\
\hline $\begin{array}{l}\text { MMI } \\
\text { Refs. [48-51] }\end{array}$ & $\begin{array}{l}\text { Small size } \\
\text { Faster response } \\
\text { Large operating frequency bandwidth } \\
\text { Low power operation }\end{array}$ & $\begin{array}{l}\text { Different phase for logic representation } \\
\text { Input phase requirement }\end{array}$ & $\begin{array}{l}\text { Splitters } \\
\text { Demultiplexers Signal processing } \\
\text { Extreme environments sensors } \\
\text { Frequency shifters }\end{array}$ \\
\hline $\begin{array}{l}\text { Waveguide Interference Paths } \\
\text { Refs. [27, 28,52-55] }\end{array}$ & $\begin{array}{l}\text { Wide operating frequency bandwidth } \\
\text { Low power operation } \\
\text { Fast response } \\
\text { Reasonable contrast ratio }\end{array}$ & $\begin{array}{l}\text { Large size } \\
\text { Difficult to synchronize the phase difference }\end{array}$ & $\begin{array}{l}\text { Signal processing } \\
\text { Routing } \\
\text { Parallel computing } \\
\text { Hybrid system integration }\end{array}$ \\
\hline $\begin{array}{l}\text { Nonlinear Effects } \\
\text { Refs. }[22,31,56-58,60]\end{array}$ & $\begin{array}{l}\text { High contrast ratio } \\
\text { Input phase independent }\end{array}$ & $\begin{array}{l}\text { Narrow operating frequency bandwidth } \\
\text { High power consumption } \\
\text { Slow response }\end{array}$ & $\begin{array}{l}\text { Sensors } \\
\text { Signal processing } \\
\text { Switching } \\
\text { Integrated photonics devices } \\
\text { Logic circuits }\end{array}$ \\
\hline
\end{tabular}

Table I.: Summary of the advanced, weakness and possible applications for each PhC logic gates approach.

power operation, fast response, and reasonable contrast ratio. They are simple designs for two and three inputs logic gates showing high availability and fault tolerance. They can also operate within the entire bandgap of the PhC. Their input power independence also allows the operation in the low power regime with low losses and slight heat dissipation. In contrast, their size typically is in the order of few microns above the operating wavelength. Also, logic gates up to three inputs are challenging to achieve since it is difficult to synchronize and control the phase difference for the light input signals. This fact can also latch their microfabrication. In addition, the feedback propagation to the inactive inputs has limited their application for large scale circuits. In particular, these kinds of devices can be extensively used for applications in signal processing, routing, parallel computing, and hybrid system integration.

Nonlinear PhC logic gates can reach high contrast ratios and are input phase independent. LG based on this approach can have high power operation due to the required optical control pulse to generate the nonlinear effects. Also, the response time and the bandwidth are limited by the nonlinear material and the resonant frequency of the resonator, respectively. LG based on this approach are good candidates for integrated photonic devices, sensors, switching, and signal processing. They can also be applied towards the realization of PhC logic circuits.

Overall, the development of $\mathrm{PhC}$ logic gates has gain attention in recent years due to the importance of these systems to build the future of the fully photonic communication and computer systems. Here, we have evaluated 139 papers related to this subject retrieved from IEEE, IET, AIP, OSA, Elsevier, IOP, Springer. These are summarized in Figure 19. This fact is evident with the increasing number of papers published per years (see Figure 19a), compiled on $75 \%$ of journal articles and $25 \%$ proceedings and conference meetings. As expected, we found that the most used logic gates to build circuits appears at the top of the implementations, as illustrated in Figure 19b. On the other hand, Figure 19c shows that logic gates are implemented on a $\mathrm{PhC}$ lattice con- figuration of rods on air and holes in a substrate with $83 \%$ and $16 \%$, respectively. It can explain the poor number of experimental works $(3 \%)$ since the former still a challenging fabrication process. Finally, we found that employed materials are the most common in integrated photonic technology, such as $\mathrm{Si}, \mathrm{GaAs}, \mathrm{SiO}_{2}$, AlGaAs, InP, Ag-Polymer, Lithium Niobate, Germanio and Chalcogenide glass, as illustrated in Figure 19d.

These achievements allow us to address and take advantage of $\mathrm{PhC}$ logic gates for a wide range of integrated photonics technology applications. However, the demonstration of PhC circuits still remains a big challenge rather than simple or compact isolated logic gates. It is also necessary to develop and improve fabrication processes to implement different $\mathrm{PhC}$ logic devices based on each technique. Another important point to consider is the standardization design for each method exploited here. Within the same approach, different dimensions, unequal transmission, or power values representing the same logic value can generate undesirable behaviors of devices.

\section{Conclusion}

We presented here a literature review on different approaches to build $\mathrm{PhC}$ logic devices. We found that $\mathrm{PhC}$ logic gates have been successfully demonstrated using selfcollimation effect, MMI, waveguide interference paths and nonlinear effects.

We highlight that each approach is suitable for particular applications considering its advantages and weakness. For instance, SC and MMI devices can be used for compactness and small devices, and for phase shifters and modulators. Logic gates based on waveguide interference paths are useful to design photonic systems that requires low power operation and fast response time. Finally, nonlinear $\mathrm{PhC}$ logic gates can play an important role to build devices with high contrast ratio towards the development of integrated circuits.

With this review and with the considerations presented here, we expect to improve the development of $\mathrm{PhC}$ integrated logic devices and circuits. 

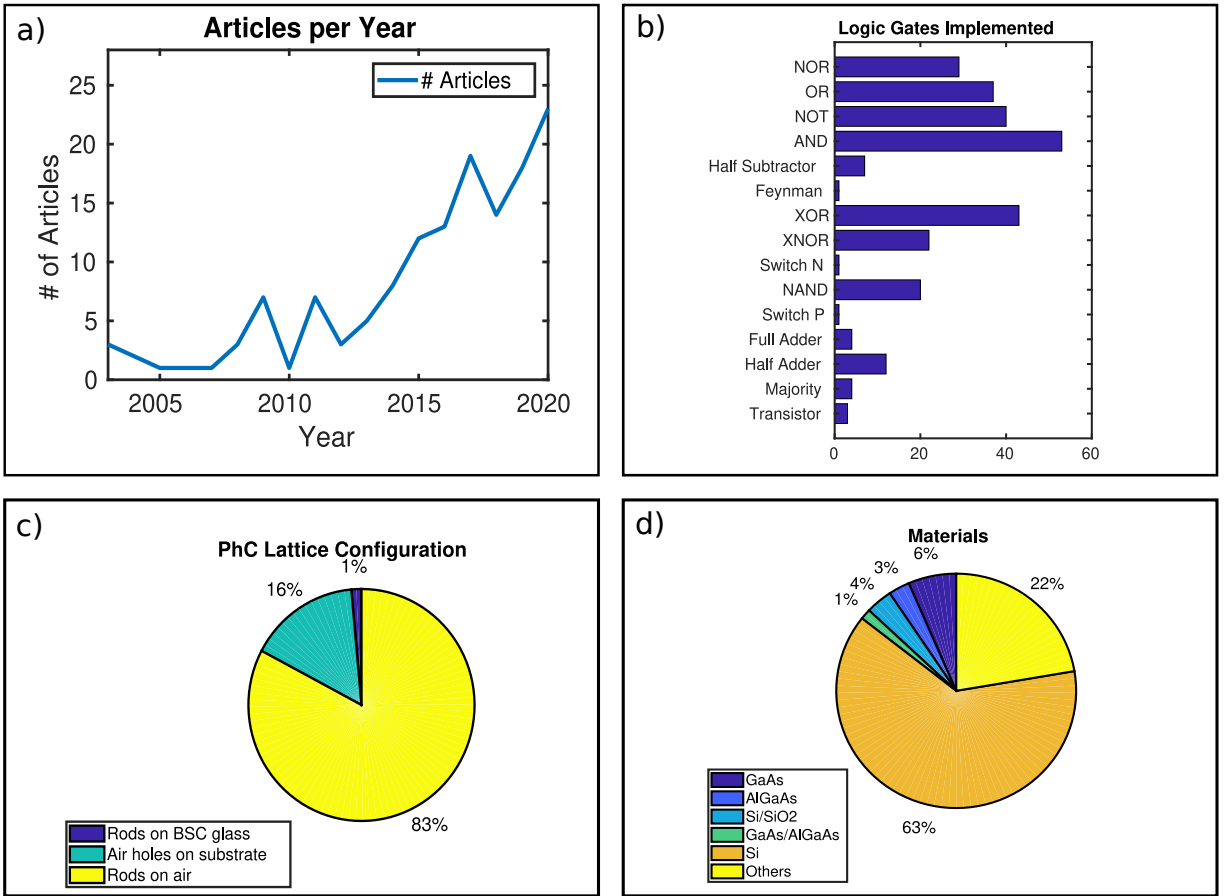

Fig. 19: Summary of the photonic crystal logic gates literature review. a) Articles published per year. b) Number of logic gates implemented. c) PhC lattice configurations and d) materials used to design logic gates.

\section{REFERENCES}

[1] E. Yablonovitch, "Inhibited spontaneous emission in solid-state physics and electronics," Physical review letters, vol. 58, no. 20, p. 2059, 1987.

[2] S. John, "Strong localization of photons in certain disordered dielectric superlattices," Physical review letters, vol. 58, no. 23, p. 2486, 1987.

[3] E. Yablonovitch, T. Gmitter, and K.-M. Leung, "Photonic band structure: The face-centered-cubic case employing nonspherical atoms," Physical review letters, vol. 67, no. 17, p. 2295, 1991.

[4] S. E. Baker, M. D. Pocha, A. S. Chang, D. J. Sirbuly, S. Cabrini, S. D. Dhuey, T. C. Bond, and S. E. Létant, "Detection of bio-organism simulants using random binding on a defect-free photonic crystal," Applied Physics Letters, vol. 97, no. 11, p. 113701, 2010.

[5] D. Englund, A. Majumdar, A. Faraon, M. Toishi, N. Stoltz, P. Petroff, and J. Vučković, "Resonant excitation of a quantum dot strongly coupled to a photonic crystal nanocavity," Physical Review Letters, vol. 104, no. 7, p. 073904, 2010.

[6] J. Knight, T. Birks, P. S. J. Russell, and D. Atkin, "All-silica singlemode optical fiber with photonic crystal cladding," Optics Letters, vol. 21, no. 19, pp. 1547-1549, 1996.

[7] N. Skivesen, A. Têtu, M. Kristensen, J. Kjems, L. H. Frandsen, and P. I. Borel, "Photonic-crystal waveguide biosensor," Optics Express, vol. 15, no. 6, pp. 3169-3176, 2007.

[8] M. Notomi, K. Yamada, A. Shinya, J. Takahashi, C. Takahashi, and I. Yokohama, "Extremely large group-velocity dispersion of linedefect waveguides in photonic crystal slabs," Physical Review Letters, vol. 87 , no. 25 , p. $253902,2001$.

[9] I. A. Sukhoivanov and I. V. Guryev, Photonic crystals: physics and practical modeling. Springer, 2009, vol. 152.

[10] J. Joannopoulos, S. Johnson, J. Winn, and R. Meade, Photonic Crystals: Molding the Flow of Light - Second Edition. Princeton University Press, 2011.
[11] J. D. Joannopoulos, P. R. Villeneuve, and S. Fan, "Photonic crystals: putting a new twist on light," Nature, vol. 386, no. 6621, p. 143, 1997.

[12] S. Takahashi, K. Suzuki, M. Okano, M. Imada, T. Nakamori, Y. Ota, K. Ishizaki, and S. Noda, "Direct creation of three-dimensional photonic crystals by a top-down approach," Nature materials, vol. 8, no. 9 , p. $721,2009$.

[13] E. C. Nelson, N. L. Dias, K. P. Bassett, S. N. Dunham, V. Verma, M. Miyake, P. Wiltzius, J. A. Rogers, J. J. Coleman, X. Li et al., "Epitaxial growth of three-dimensionally architectured optoelectronic devices," Nature materials, vol. 10, no. 9, p. 676, 2011.

[14] K. Ishizaki, M. Koumura, K. Suzuki, K. Gondaira, and S. Noda, "Realization of three-dimensional guiding of photons in photonic crystals," Nature Photonics, vol. 7, no. 2, p. 133, 2013.

[15] S. G. Johnson, S. Fan, P. R. Villeneuve, J. D. Joannopoulos, and L. Kolodziejski, "Guided modes in photonic crystal slabs," Physical Review B, vol. 60, no. 8, p. 5751, 1999.

[16] P. R. Villeneuve, S. Fan, and J. Joannopoulos, "Microcavities in photonic crystals: Mode symmetry, tunability, and coupling efficiency," Physical Review B, vol. 54, no. 11, p. 7837, 1996.

[17] Y. Akahane, T. Asano, B.-S. Song, and S. Noda, "High-q photonic nanocavity in a two-dimensional photonic crystal," nature, vol. 425, no. 6961, p. 944, 2003.

[18] T. Asano, B.-S. Song, Y. Tanaka, and S. Noda, "Investigation of a channel-add/drop-filtering device using acceptor-type point defects in a two-dimensional photonic-crystal slab," Applied physics letters, vol. 83, no. 3, pp. 407-409, 2003.

[19] T. Asano, M. Mochizuki, S. Noda, M. Okano, and M. Imada, "A channel drop filter using a single defect in a 2-d photonic crystal slab-defect engineering with respect to polarization mode and ratio of emissions from upper and lower sides," Journal of lightwave technology, vol. 21, no. 5 , p. 1370,2003 .

[20] C. Seassal, Y. Dsieres, X. Letartre, C. Grillet, P. Rojo-Romeo, P. Viktorovitch, and T. Benyattou, "Optical coupling between a twodimensional photonic crystal-based microcavity and single-line defect 
waveguide on inp membranes," IEEE Journal of quantum electronics, vol. 38, no. 7, pp. 811-815, 2002.

[21] B.-K. Min, J.-E. Kim, and H. Y. Park, "Channel drop filters using resonant tunneling processes in two-dimensional triangular lattice photonic crystal slabs," Optics Communications, vol. 237, no. 1-3, pp. $59-63,2004$.

[22] M. F. Yanik, S. Fan, and M. Soljačić, "High-contrast all-optical bistable switching in photonic crystal microcavities," Applied Physics Letters, vol. 83, no. 14, pp. 2739-2741, 2003.

[23] E. Waks and J. Vuckovic, "Coupled mode theory for photonic crystal cavity-waveguide interaction," Optics Express, vol. 13, no. 13, pp. 5064-5073, 2005 .

[24] J. Bravo-Abad, A. Rodriguez, P. Bermel, S. G. Johnson, J. D. Joannopoulos, and M. Soljačić, "Enhanced nonlinear optics in photonic-crystal microcavities," Optics express, vol. 15, no. 24, pp. 16 161-16176, 2007.

[25] L. B. Soldano and E. C. Pennings, "Optical multi-mode interference devices based on self-imaging: principles and applications," Journal of lightwave technology, vol. 13, no. 4, pp. 615-627, 1995.

[26] D. W. Prather, S. Shi, J. Murakowski, G. J. Schneider, A. Sharkawy, C. Chen, B. Miao, and R. Martin, "Self-collimation in photonic crystal structures: a new paradigm for applications and device development," Journal of Physics D: Applied Physics, vol. 40, no. 9, p. 2635, 2007.

[27] Y. Fu, X. Hu, and Q. Gong, "Silicon photonic crystal all-optical logic gates," Physics letters A, vol. 377, no. 3-4, pp. 329-333, 2013.

[28] N. M. D'souza and V. Mathew, "Interference based square lattice photonic crystal logic gates working with different wavelengths," Optics \& Laser Technology, vol. 80, pp. 214-219, 2016.

[29] R. W. Boyd, Nonlinear optics. Elsevier, 2003.

[30] E. Kuramochi, K. Nozaki, A. Shinya, H. Taniyama, K. Takeda, T. Sato, S. Matsuo, and M. Notomi, "Ultralow bias power alloptical photonic crystal memory realized with systematically tuned 13 nanocavity," Applied Physics Letters, vol. 107, no. 22, p. 221101, 2015.

[31] M. F. Yanik, S. Fan, M. Soljačić, and J. D. Joannopoulos, "All-optical transistor action with bistable switching in a photonic crystal crosswaveguide geometry," Optics letters, vol. 28, no. 24, pp. 2506-2508, 2003.

[32] J. Zhou, H. Shao, J. Zhao, X. Yu, and K. Wong, "Storage and release of femtosecond laser pulses in a resonant photonic crystal," Optics letters, vol. 30, no. 12, pp. 1560-1562, 2005.

[33] B. Z. Steinberg and A. Boag, "Propagation in photonic crystal coupled-cavity waveguides with discontinuities in their optical properties," JOSA B, vol. 23, no. 7, pp. 1442-1450, 2006.

[34] Z.-H. Zhu, W.-M. Ye, J.-R. Ji, X.-D. Yuan, and C. Zen, "High-contrast light-by-light switching and and gate based on nonlinear photonic crystals," Optics Express, vol. 14, no. 5, pp. 1783-1788, 2006.

[35] S. Sandhu, M. Povinelli, and S. Fan, "Stopping and time reversing a light pulse using dynamic loss tuning of coupled-resonator delay lines," Optics letters, vol. 32, no. 22, pp. 3333-3335, 2007.

[36] D. G. Angelakis, M. F. Santos, V. Yannopapas, and A. Ekert, "A proposal for the implementation of quantum gates with photonic-crystal waveguides," Physics Letters A, vol. 362, no. 5-6, pp. 377-380, 2007.

[37] A. Shinya, S. Matsuo, T. Tanabe, E. Kuramochi, T. Sato, T. Kakitsuka, M. Notomi et al., "All-optical on-chip bit memory based on ultra high q ingaasp photonic crystal," Optics express, vol. 16, no. 23, pp. 19382-19387, 2008.
[38] A. Taflove and S. C. Hagness, Computational electrodynamics: the finite-difference time-domain method. Artech house, 2005.

[39] D. M. Sullivan, Electromagnetic simulation using the FDTD method. John Wiley \& Sons, 2013.

[40] J. B. Schneider, "Understanding the finite-difference time-domain method," School of electrical engineering and computer science Washington State University., 2010.

[41] K.-M. Leung and Y. Liu, "Photon band structures: The plane-wave method," Physical Review B, vol. 41, no. 14, p. 10188, 1990.

[42] S. G. Johnson and J. D. Joannopoulos, "Block-iterative frequencydomain methods for maxwell's equations in a planewave basis," $O P$ tics express, vol. 8, no. 3, pp. 173-190, 2001.

[43] S. Guo and S. Albin, "Simple plane wave implementation for photonic crystal calculations," Optics Express, vol. 11, no. 2, pp. 167-175, 2003.

[44] Y. Zhang, Y. Zhang, and B. Li, "Optical switches and logic gates based on self-collimated beams in two-dimensional photonic crystals," $\mathrm{Op}$ tics Express, vol. 15, no. 15, pp. 9287-9292, 2007.

[45] X. S. Christina and A. Kabilan, "Design of optical logic gates using self-collimated beams in 2d photonic crystal," Photonic Sensors, vol. 2, no. 2, pp. 173-179, 2012.

[46] R. Fan, X. Yang, X. Meng, and X. Sun, "2d photonic crystal logic gates based on self-collimated effect," Journal of Physics D: Applied Physics, vol. 49, no. 32, p. 325104, 2016.

[47] M. R. Jalali-Azizpoor, M. Soroosh, and Y. Seifi-Kavian, "Application of self-collimated beams in realizing all-optical photonic crystal-based half-adder," Photonic Network Communications, vol. 36, no. 3, pp. 344-349, 2018.

[48] Y. Ishizaka, Y. Kawaguchi, K. Saitoh, and M. Koshiba, "Design of ultra compact all-optical xor and and logic gates with low power consumption," Optics Communications, vol. 284, no. 14, pp. 3528-3533, 2011.

[49] W. Liu, D. Yang, G. Shen, H. Tian, and Y. Ji, "Design of ultra compact all-optical xor, xnor, nand and or gates using photonic crystal multimode interference waveguides," Optics \& Laser Technology, vol. 50, pp. 55-64, 2013.

[50] C. Tang, X. Dou, Y. Lin, H. Yin, B. Wu, and Q. Zhao, "Design of all-optical logic gates avoiding external phase shifters in a twodimensional photonic crystal based on multi-mode interference for bpsk signals," optics Communications, vol. 316, pp. 49-55, 2014.

[51] E. haq Shaik and N. Rangaswamy, "Multi-mode interference-based photonic crystal logic gates with simple structure and improved contrast ratio," Photonic Network Communications, vol. 34, no. 1, pp. 140-148, 2017.

[52] L. P. Caballero, J. Vasco, P. Guimaraes, and O. P. V. Neto, "All-optical majority and feynman gates in photonic crystals," in 2015 30th Symposium on Microelectronics Technology and Devices (SBMicro). IEEE, 2015, pp. 1-4.

[53] L. E. P. Caballero, J. P. V. Cano, P. S. Guimarães, and O. P. V. Neto, "Effect of structural disorder on photonic crystal logic gates," IEEE Photonics Journal, vol. 9, no. 5, pp. 1-15, 2017.

[54] H. M. Hussein, T. A. Ali, and N. H. Rafat, "New designs of a complete set of photonic crystals logic gates," Optics Communications, vol. 411, pp. $175-181,2018$.

[55] L. P. Caballero, M. Povinelli, J. C. Ramirez, P. Guimaraes, and O. P. V. Neto, "Design of compact integrated photonic crystal nand and nor logic gates," in 2020 23rd Euromicro Conference on Digital System Design (DSD). IEEE, 2020, pp. 420-427. 
[56] M. Neisy, M. Soroosh, and K. Ansari-Asl, "All optical half adder based on photonic crystal resonant cavities," Photonic Network Communications, vol. 35, no. 2, pp. 245-250, 2018.

[57] H. Alipour-Banaei and H. Seif-Dargahi, "Photonic crystal based 1bit full-adder optical circuit by using ring resonators in a nonlinear structure," Photonics and Nanostructures - Fundamentals and Applications, vol. 24, pp. 29 - 34, 2017.

[58] M. Hassangholizadeh-Kashtiban, H. Alipour-Banaei, M. B. Tavakoli, and R. Sabbaghi-Nadooshan, "An ultra fast optical reversible gate based on electromagnetic scattering in nonlinear photonic crystal resonant cavities," Optical Materials, vol. 94, pp. 371 - 377, 2019.

[59] R. Landauer, "Irreversibility and heat generation in the computing process," IBM Journal of Research and Development, vol. 5, no. 3, pp. 183-191, July 1961.

[60] L. P. Caballero, M. L. Povinelli, J. C. Ramirez, P. S. Guimarães, and O. P. V. Neto, "Complementary photonic crystal integrated logic devices," Optics Letters, vol. 45, no. 19, pp. 5502-5505, 2020.

[61] A. Coelho Jr, M. Costa, A. Ferreira, M. Da Silva, M. Lyra, and A. Sombra, "Realization of all-optical logic gates in a triangular triplecore photonic crystal fiber," Journal of lightwave technology, vol. 31, no. 5, pp. 731-739, 2013.

[62] L. He, W. Zhang, and X. Zhang, "Topological all-optical logic gates based on two-dimensional photonic crystals," Optics express, vol. 27, no. 18 , pp. $25841-25859,2019$.

[63] A. Kotb and C. Guo, "Numerical modeling of photonic crystal semiconductor optical amplifiers-based $160 \mathrm{gb} / \mathrm{s}$ all-optical nor and xnor logic gates," Optical and Quantum Electronics, vol. 52, no. 2, p. 89, 2020 .

[64] S. Kumar and M. Sen, "Integrable all-optical not gate using nonlinear photonic crystal mzi for photonic integrated circuit," JOSA B, vol. 37 , no. 2, pp. 359-369, 2020.

[65] T. Datta and M. Sen, "Raman mediated ultrafast all-optical nor gate," Applied Optics, vol. 59, no. 21, pp. 6352-6359, 2020. 